\title{
The halo-to-stellar mass ratio in the $\mathrm{S}^{4} \mathrm{G}$
}

\section{Simón Díaz-García, Heikki Salo and Eija Laurikainen}

Astronomy Research Group, University of Oulu, FI-90014 Finland, email: simon.diazgarcia@oulu.fi

\begin{abstract}
We use $3.6 \mu \mathrm{m}$ photometry for 1154 disk galaxies $\left(i<65^{\circ}\right)$ in the $\mathrm{S}^{4} \mathrm{G}$ (Sheth et al. $2010)$. We obtain the average stellar component of the circular velocity $\left(V_{\text {disk }}\right)$ and the mean (dark matter) halo-to-stellar mass ratio $\left(M_{\mathrm{halo}} / M_{*}\right)$ inside the optical radius $\left(R_{\mathrm{opt}}\right)$ in bins of total stellar mass $\left(M_{*}\right.$, from Muñoz-Mateos et al. 2015), providing observational constraints for galaxy formation models to be tested against. We find the $M_{\mathrm{halo}} / M_{*}-M_{*}$ relation in good agreement with the best-fit model at $\mathrm{z} \approx 0$ in $\Lambda \mathrm{CDM}$ cosmological simulations (e.g. Moster 2010), assuming that the dark matter halo within $R_{\mathrm{opt}}$ comprises a constant fraction $(\sim 4 \%)$ of its total mass.
\end{abstract}

Keywords. galaxies: structure - galaxies: dark matter - galaxies: statistics

We obtain the mean $V_{\text {disk }}$ and $M_{\text {halo }} / M_{*}\left(<R_{\text {opt }}\right)$ in bins of $M_{*}$ (Fig. 1$)$ using the gravitational potentials $(\Phi)$ of $\mathrm{S}^{4} \mathrm{G}$ disk galaxies (Díaz-García et al. 2016):

$V_{\mathrm{disk}}(r)=\sqrt{\Upsilon_{3.6 \mu \mathrm{m}}\left\langle\frac{\partial \Phi}{\partial r}(r)\right\rangle r} \quad \& \quad M_{\mathrm{halo}} / M_{*}\left(<R_{\mathrm{opt}}\right) \approx 1.34 \cdot\left(\frac{\left(V_{\mathrm{HI}}^{\max }\right)^{2}}{V_{\mathrm{disk}}^{2}\left(R_{\mathrm{opt}}\right)}-1\right)$, where $\Upsilon_{3.6 \mu \mathrm{m}}=0.53$ is the mass-to-light ratio at $3.6 \mu \mathrm{m}$ (Eskew et al. 2012), $R_{\text {opt }} \approx$ $3.2 \cdot\left\langle h_{\mathrm{R}}\right\rangle\left(h_{\mathrm{R}}\right.$ being the disk scalelength from Salo et al. 2015) and $V_{\mathrm{HI}}^{\mathrm{max}}$ is the mean inclination-corrected $\mathrm{H}$ I line width from the literature (e.g. Courtois et al. 2011).
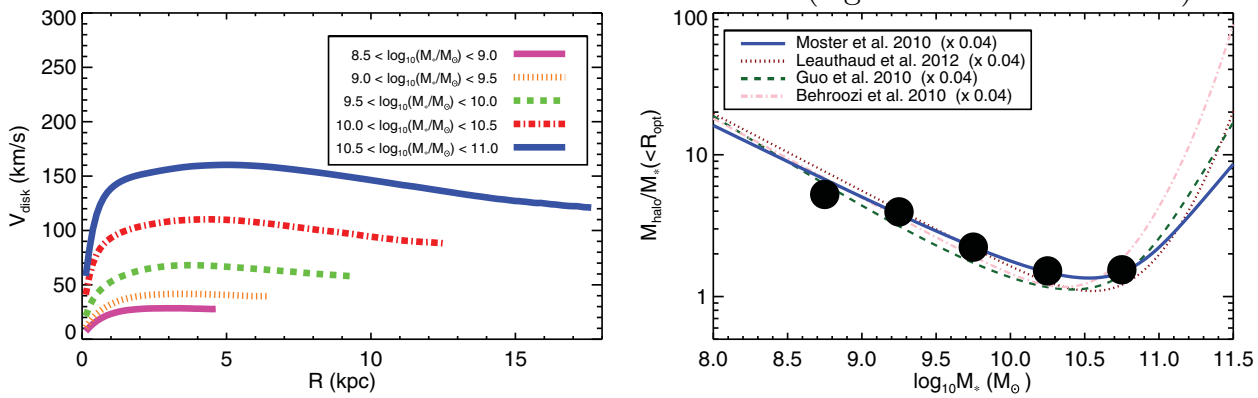

Figure 1. Left panel: Mean $V_{\text {disk }}$ for the different $M_{*}$-bins. Right panel: The central value of the $M_{*}$-bins vs. the mean $M_{\text {halo }} / M_{*}\left(<R_{\mathrm{opt}}\right)$ (filled circles). The dashed lines correspond to estimates in the literature for the total halo-to-stellar mass ratio vs. $M_{*}$, scaled down by a factor 0.04, showing good agreement with our estimate (Díaz-García et al. 2016).

We acknowledge financial support to the DAGAL network from the People Programme (Marie Curie Actions) of the European Union's Seventh Framework Programme FP7/20072013/ under REA grant agreement number PITN-GA-2011-289313. We thank Ryan Leaman for very useful discussion. We thank the organizers of the IAU 321 symposium.

\section{References}

Courtois, H. M., Tully, R. B., Makarov, D. I., et al. 2011, MNRAS, 414, 2005

Díaz-García, S., Salo, H., Laurikainen, E., \& Herrera-Endoqui, M. 2016, A\&AA, 587, A160

Eskew, M., Zaritsky, D., \& Meidt, S. 2012, AJ, 143, 139

Moster, B. P., Somerville, R. S., Maulbetsch, C., et al. 2010, ApJ, 710, 903

Muñoz-Mateos, J. C., Sheth, K., Regan, M., et al. 2015, ApJS, 219, 3

Salo, H., Laurikainen, E., Laine, J., et al. 2015, ApJS, 219, 4

Sheth, K., Regan, M., Hinz, J. L., et al. 2010, PASP, 122, 1397 